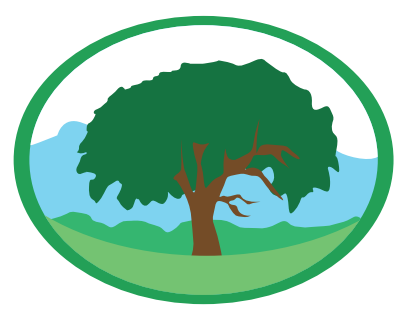

\title{
Incidência de cânceres e o uso de agrotóxicos na região oeste do Paraná
}

\author{
NEGE, K. K. ${ }^{1}$; NADALETI, W. C. ${ }^{2}$; BORTOLOTTI, S. L. V. ${ }^{3}$; SILVA, R. F. ${ }^{4}$; FAVARETTO, A. P. ${ }^{5}$; MANZINI, V. ${ }^{6}$ \\ ${ }^{1}$ Acadêmico de engenharia de petróleo da Universidade Federal Fluminense - UFF/Niterói, kassemknegelahotmail.com; \\ ${ }^{2}$ Professor do Centro de Engenharias da Universidade Federal de Pelotas - UFPel, willian.nadaletiQufpel.edu.br; \\ ${ }^{3}$ Núcleo de Matemática e Estatística/UTFPR-Medianeira, sligielaglobo.com; \\ 4,5,6Acadêmicos de Engenharia de Produção UTFPR-Medianeira; \\ ${ }^{4}$ rodrigo_f_digolahotmail.com; \\ 5apaulafavarettodgmail.com; \\ ${ }^{6}$ vinimanziniahotmail.com
}

Palavras-chave: saúde pública, câncer, estatística espacial, agrotóxicos

\section{Resumo}

Recentemente vários estudos estão sendo realizados para verificar o potencial carcinogênico dos agrotóxicos. Com isso, este estudo objetivou em identificar, coletar, examinar e avaliar informações obtidas por meio da aplicação de questionário, junto ao uso da estatística descritiva bem como da estatística espacial sobre o potencial carcinogênico dos agrotóxicos utilizados na região oeste do Paraná. No total foram entrevistados 162 pacientes diagnosticados com câncer, sendo que a maioria destes $(31,5 \%)$ residiam no município de CascavelPR. De modo geral, mais de $50 \%$ dos entrevistados relataram ter contato com agrotóxicos, demonstrando assim, uma problemática em que possivelmente o uso de agrotóxicos possa estar relacionado como uma das causas de câncer na população. Com base nisto, este estudo propõem a criação de programas públicos voltados para os agricultores com o intuito de oferecer alternativas e apoio para que esses consigam reformular o seu processo de produção, substituindo o modo convencional de produção agrícola, por práticas de produção alternativas, como as práticas agroecológicas.

\section{Cancer incidence and use of pesticides in the west region of Paraná}

Keywords: public health, cancer, spatial statistics, pesticides

\begin{abstract}
Recently several studies have been conducted to evaluate carcinogenic potential of pesticides. Therefore, this study aimed to identify, collect, examine and evaluate information obtained through questionnaires, with the use of descriptive statistics and spatial statistics about the carcinogenic potential of pesticides used in western Paraná. In total we interviewed 162 patients diagnosed with cancer, and the majority of these (31.5\%) lived in Cascavel-PR. Overall, more than $50 \%$ of respondents reported having contact with pesticides, thus demonstrating an issue that possibly the use of pesticides may be related as a cause of cancer in the population. On this basis, this paper proposes the creation of public programs for farmers in order to provide alternatives and support for those able to reshape your production process by replacing the conventional mode of agricultural production, alternative production practices, such as agroecological practices.
\end{abstract}




\section{INTRODUC̣ÃO}

O uso de agrotóxicos na agricultura brasileira começou a partir da década de 60 , por meio do Plano Nacional do Desenvolvimento (PND), o qual esse concedia créditos rurais aos agricultores que comprassem uma cota definida de agrotóxicos. Com isso, a utilização desses produtos aumentou de forma exponencial, extinguindo quase a totalidade de práticas alternativas e sustentáveis na produção agrícola (GARCIA, 1996).

O principal motivo da utilização dos agrotóxicos no mundo é devido ao aumento de produtividade, pois esse garante o sucesso da safra ao estabelecer o controle das pragas e doenças que se estabelecem nas culturas. Possibilitando assim, o abastecimento da grande demanda de alimentos da população bem como o sucesso do agronegócio (VEIGA, 2007).

O Brasil conquistou em 2009 o título de o país que tem o maior consumo de agrotóxicos do mundo, contradizendo a relação de produtividade/consumo, pois o Brasil não é o principal produtor agrícola mundial (BOMBARDI, 2011).

Segundo Batista Filho e Melo (2012), no Brasil são utilizados quase $5 \mathrm{~kg}$ de defensivos agrícolas por cada habitante. Tendo como consequência a contaminação de águas continentais e oceânicas, solos e plantas, animais terrestres e aquáticos e pessoas de todas as idades com os efeitos nocivos de seu manejo, inalação e ingestão. É um grave problema não local, mas sim mundial que a cada dia vem se intensificando. Esse problema náo fica concentrado somente com as pessoas que têm contato direto com esses agrotóxicos, mas também há os indivíduos que sofrem de forma indireta, pois todos nós, diariamente, ingerimos uma pequena parcela de agrotóxico, este impregnado nos nossos alimentos. Esse agrotóxico pode se acumular no nosso corpo, estando presente até no leite materno, o mais natural e o mais completo dos alimentos para as fases iniciais de um bebê.

Os impactos na saúde humana são divididos em duas formas, a forma aguda, ou seja, por intoxicação direta por agrotóxico, seja essa na hora da aplicação deste, ou então na ingestão de um alimento altamente contaminado. E a segunda forma seria pela intoxicação crônica, a longo prazo. No documento da Organização
Pan-Americana da Saúde-OPAS (1996) encontram-se as principais manifestaçóes de intoxicação crônica, entre elas há destacada diversos tipos de câncer. O câncer está dentre uma das doenças causadas pela intoxicação crônica por agrotóxicos. Segundo Koifman; Hatagima (2003), o câncer é causado por fatores externos e internos, estando ambos interrelacionados. Os fatores externos se referem às exposições ambientais enquanto os internos são, na maioria das vezes, geneticamente determinados e estáo relacionados à capacidade individual de se defender das agressóes internas. Com isso os agrotóxicos estão vinculados aos fatores externos na causa do câncer, tendo assim, um alto poder carcinogênico.

Devido a esses e vários outros problemas do uso intensivo de agrotóxicos, centros de pesquisas vêm elaborando e aplicando estudos com o intuito de investigar e propor soluçóes para evitar e mitigar os malefícios que estes agrotóxicos podem propiciar a população. De acordo com Jobim et al. (2010), é de enorme importância identificar na população os grupos mais susceptíveis, pois só assim, podem ser criados programas de saúde específicos com o intuito de prevenção dessas doenças.

Com isso este estudo tem como objetivo identificar, coletar, examinar e avaliar informaçôes obtidas por meio da estatística descritiva, bem como da estatística espacial sobre o potencial carcinogênico dos agrotóxicos utilizados na regiâo oeste do Paraná.

\section{METODOLOGIA}

O instrumento de avaliação utilizado para a coleta dos dados quantitativos e qualitativos foi por meio de um questionário. A coleta de dados foi realizada no Hospital União Oeste Paranaense de Estudos e Combate ao Câncer (UOPECCAN) no qual é referência em: prevenção, diagnóstico, tratamento por câncer e está situado no município de Cascavel, Paraná. O complexo UOPECCAN foi escolhido para estudo, pois apresenta grande número de casos de tratamento por neoplasia de entrevistados das regióes do oeste do Paraná. O questionário pode ser visualizado de acordo com a Figura 1. 
Idade:

Reside: ( ) Zona rural ( ) Urbana

Endereço atual/ Bairro/ Cidade:

Residência anterior:

Neoplasia:

Tem histórico na família de neoplasia? ( ) Sim ( ) Não

Grau de parentesco: () Pais ( ) Irmãos ( ) Avós ( ) Tios e Tias

Fumante: () Sim ( ) Não

Teve contato com agrotóxico: ( $)$ Sim( )Não

Se sim:

Cultura -

Contato: $(0)$ Direto

( ) Indireto
Figura 1. Questionário aplicado aos 162 pacientes do Hospital União Oeste Paranaense de Estudos e Combate ao Câncer (UOPECCAN)

$\mathrm{O}$ instrumento foi submetido ao comitê de ética aprovado pelo parecer $n^{\circ}$. 243.374 em 11/04/2013.

Ogrupo deestudantes junto ao orientador realizaram visitas ao hospital no mês de julho de 2013 para aplicar os questionários junto aos entrevistados. Conforme já apresentado, só foram considerados os entrevistados que residiam nos municípios do oeste do Paraná.

A partir da coleta, foi realizada a apuraçáo dos dados por meio da estatística descritiva, inferencial e espacial. Os testes e confecção dos dados foram realizados com o auxílio dos programas Bioestat 5.0 (AYRES et al., 2007) e TerraView-4.2.2 (INPE, 2010).

A estatística descritiva possibilita a descrição do perfil dos entrevistados elaborando tabelas de frequência, gráfico e tabelas de contingências. Já a estatística inferencial nos possibilita obter conclusóes sobre os dados através de hipóteses.

De acordo com Assunção (2001), a característica fundamental da estatística espacial, que se diferencia da estatística clássica, é o uso explícito da referência geográfica no modelo, isto é, o uso explícito das coordenadas espaciais no processo de coleta, descrição e análise dos dados. Assim sendo, o interesse está centrado nos processos que ocorrem no espaço e os métodos empregados buscam descrever e analisar o comportamento desses processos. Essa característica faz com que estudos sobre o assunto exibam comportamento complexo, para serem analisados por métodos tradicionais de estatística. Desta forma, a análise espacial pode ser definida como uma técnica que busca descrever os padróes existentes nos dados espaciais e estabelecer, preferencialmente de forma quantitativa, os relacionamentos entre as diversas variáveis geográficas.

\section{RESULTADOS E DISCUSSÃO}

No total foram entrevistados 162 indivíduos, sendo 83 mulheres e 79 homens. A Figura 2 apresenta a localização de residência dos entrevistados.
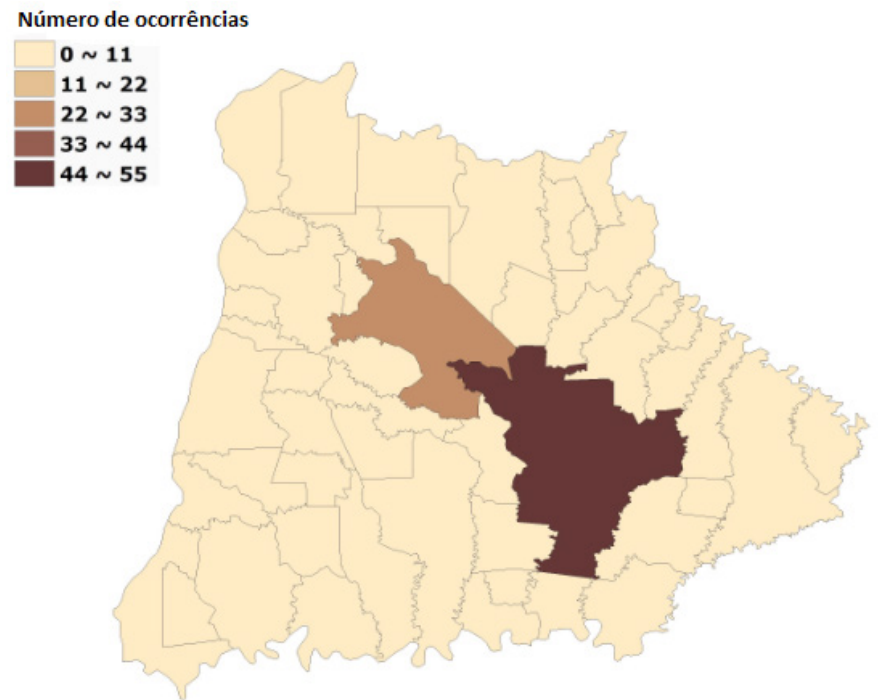

Figura 2. Mapa da região oeste do Paraná: Distribuição espacial da ocorrência de câncer

Por meio da Figura 2, visualizam-se os 50 municípios que contemplam a região oeste do Paraná, e nota-se que a maior parte dos entrevistados residem nas cidades de Cascavel (31,5\%) e Toledo (13,6\%), isso era esperado pelo fato de se encontrar a maior parte da população oeste nestes municípios, e também por ser a localização da coleta dos dados já que o hospital se localiza na cidade de Cascavel. O que não foi esperado é que o terceiro município com a maior ocorrência de neoplasia fosse Missal (4,9\%), sendo que este é um pequeno município em comparaçáo com os demais, segundo o último censo nele se encontra 10.474 habitantes (IBGE, 2010).

A Figura 3 apresenta a distribuição dos entrevistados que relataram ter contato com agrotóxicos (57\% dos casos) ao longo da região oeste do Paraná. Comparando a Figura 3 com a Figura 1, nota-se que algumas outras cidades se destacam como Missal, Terra Roxa e Marechal Cândido Rondon. Sendo que em Missal ocorreu um caso diferenciado, onde 75\% dos entrevistados tiveram contato com agrotóxico e dentre esses, $62,5 \%$ relataram o contato com agrotóxicos aplicados à cultura do fumo/tabaco. Etges (2002) evidenciou que os agrotóxicos aplicados no cultivo do tabaco, se associam significativamente com 
as intoxicaçóes e distúrbios neurocomportamentais nos produtores da folha de tabaco. Vários estudos já relataram também os problemas de saúde que o cultivo do tabaco pode causar (OLIVEIRA et al., 2010; FASSA et al., 2014; RIQUINHO; HENNINGTON, 2014).

Outro fato evidenciado através da análise da Figura 3 é a maior prevalência da utilização de agrotóxicos por residentes da zona rural (74\% dos casos), o mesmo foi evidenciado por Silva et al. (2013), pelo fato da elevada atuação destes na lavoura em relação aos quais residem na zona urbana.

Verificou-se também, que as pessoas que indicaram ter contato com agrotóxicos, a cultura de soja foi a mais citada, em torno de $57 \%$ dos relatos. A cultivação da soja é a qual consome o maior volume de agrotóxicos no Brasil. (CARNEIRO et al., 2012). Silva et al. (2015) estabeleceu em seus estudos uma correlação positiva entre toneladas de soja plantada e a mortalidade por câncer de próstata.

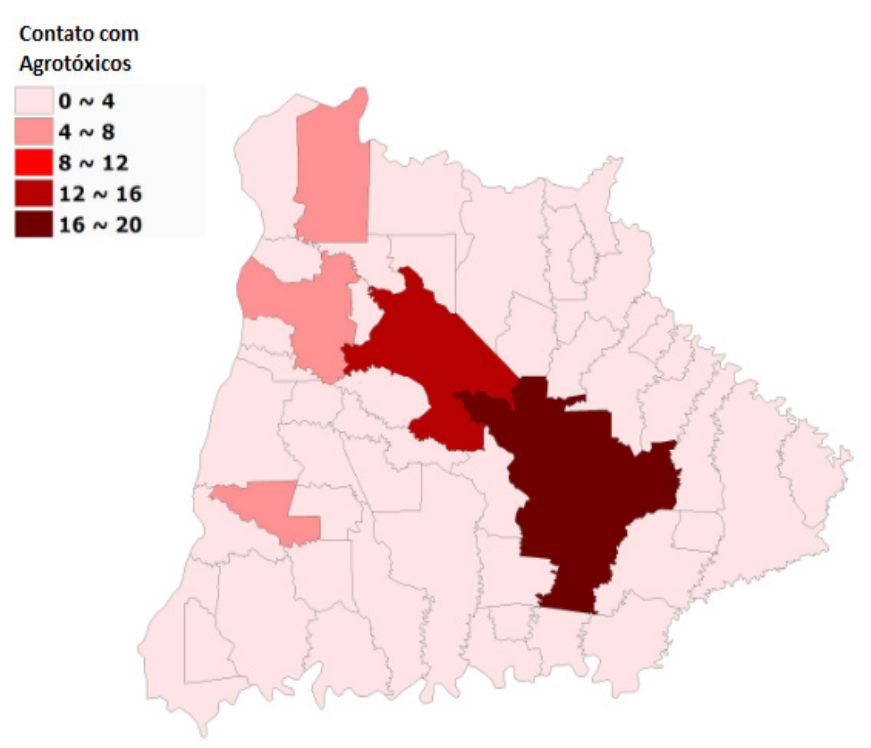

Figura 3. Mapa da região oeste do Paraná: Distribuição espacial dos entrevistados que tiveram contato com agrotóxicos

A Tabela 1 apresenta uma descrição dos entrevistados e principais tipos de câncer verificados.

Ao analisar os tipos de cânceres mais ocorridos notase, conforme a Tabela 1, que entre os entrevistados, a média de idade maior foi encontrada nos que apresentavam câncer de próstata (10\% dos casos). Observou-se também que esses tiveram um menor tempo de diagnóstico (25,1 meses) e que todos os entrevistados residiam na zona urbana. Através das médias de idades, nota-se que os entrevistados têm um perfil mais idoso, confirmando que o envelhecimento esta relacionado com as chances do desenvolvimento de câncer (FRANCESCHI, 2001). Estudos realizados por Silva et al. (2015) correlacionaram estatisticamente a mortalidade por câncer de próstata com população acima de 60 anos, e evidenciaram que há correlação desse tipo de câncer com exposição a agrotóxicos nos estados brasileiros.

Em relação aos entrevistados que possuíam o câncer de mama (17\% dos casos), observou-se que eles tiveram uma maior percentagem de contato com agrotóxicos $(57,7 \%)$, e a minoria destes foram fumantes (26,9\%). Um estudo realizado na Grécia por Dolapsakis et al. (2001) demonstrou estatisticamente a existência da relação do uso de pesticidas com alteraçóes mamográficas precursoras de câncer. Embora, vários outros estudos indicaram o contrário, não atribuindo correlação entre esses atributos (CALLE et al., 2002; COCCO, 2002; GAMMOM et al., 2002).

Já os entrevistados que possuem câncer de pele (17\% dos casos), verifica-se que estes possuem o maior índice de ocorrência de câncer na família, isso pode estar relacionado pelo fato de que seus pais ou filhos estiveram submetidos as mesmas atividades laborais, principalmente nos quais vivem na zona rural $(67 \%$ dos indivíduos da zona rural com câncer de pele relataram que seus pais, irmáos e tias também tiverem algum câncer). As atividades laborais podem estar relacionadas com o câncer de pele, principalmente no trabalho rural, pois as condiçôes de exposição à radiação solar ultravioleta e aos pesticidas são fatores de riscos que podem provocar o desenvolvimento dessa neoplasia, algumas pesquisas evidenciaram essas relaçóes (BAUER et al., 2011; DENNIS et al., 2010; LANSBURY; BATH-HEXTALL, 2012).

$\mathrm{Na}$ Tabela 2, temos a distribuição da frequência entre o contato com o agrotóxico e gênero.

Tabela 2. Distribuição da frequência entre contato com agrotóxicos e gênero

$\mathrm{O}$ valor de $p$ do teste estatístico do Qui-quadrado não foi significativo $(>0,05)$, logo se pode inferir que as frequências observadas não são diferentes das frequências esperadas para as variáveis: contato com agrotóxico e gênero, ou seja, não há associação entre 
Tabela 1. Análise dos principais tipos de câncer verificados

\begin{tabular}{|l|l|l|l|}
\hline Atributos/Tipo de Câncer & \multicolumn{1}{|c|}{ Pele (17\%) } & \multicolumn{1}{|c|}{ Mama (17\%) } & Próstata (10\%) \\
\hline Idade (anos) & $64,2 \pm 16,3$ & $53,8 \pm 10,6$ & $66,3 \pm 18,7$ \\
\hline Tempo diagnosticado (meses) & $42,7 \pm 72,0$ & $28,9 \pm 28,7$ & $25,1 \pm 30,3$ \\
\hline Contato com Agrotóxicos & $50,0 \%$ & $57,7 \%$ & $56,25 \%$ \\
\hline Fumantes & $46,4 \%$ & $26,9 \%$ & $50,0 \%$ \\
\hline Histórico na Família & $60,7 \%$ & $57,7 \%$ & $31,25 \%$ \\
\hline Zona Rural & $35,7 \%$ & $34,6 \%$ & $0,0 \%$ \\
\hline Zona Urbana & $64,3 \%$ & $65,4 \%$ & $100,0 \%$ \\
\hline Gênero Masculino & $60,7 \%$ & $3,9 \%$ & $100,0 \%$ \\
\hline Gênero Feminino & $39,3 \%$ & $96,1 \%$ & $0,0 \%$ \\
\hline
\end{tabular}

elas. Farias et al. (2003) avaliou por delineamento transversal a população rural da Serra Gaúcha chegando à conclusão que não há correlação entre gênero e o contato com agrotóxicos. Já os estudos realizados em Nova Friburgo/RJ por Moreira et al. (2002) e na regiáo Sul do Brasil por Souza et al. (2011) evidenciaram o contrário, que o trabalho rural era exercido principalmente pelo homem e esse consequentemente teria um maior contato com agrotóxico.

\section{CONCLUSÕES}

Neste trabalho observou-se o grave problema que os agrotóxicos podem causar na saúde humana, pois em todos os casos, mais de 50\% dos entrevistados diagnosticados com câncer relataram que tiveram contato com agrotóxicos. Sendo que no município de Missal verificou-se um alto número de ocorrência de câncer em uma pequena parcela da populaçáo em comparação com toda regiáo oeste, sendo que a maioria dos indivíduos relatou que já exerceram atividades laborais com o cultivo da folha do fumo. Sendo assim, sugere-se que sejam realizados novos estudos no município de Missal, abrangendo uma investigação da saúde ocupacional do produtor de fumo, buscando maiores explicaçôes para tal fato.

Portanto, para assegurar uma qualidade de vida para a populaçáo, deve-se por parte do governo, haver o incentivo e criação de novos programas com um caráter diferenciado, onde o foco principal seja o incentivo da produção sustentável, inovação na agroecologia, bem como a produção de produtos orgânicos. E sem dúvida a disseminação da educação ambiental com ênfase nos pequenos produtores.

\section{REFERÊNCIAS}

ASSUNÇĀO, R. Estatística espacial com aplicaçóes em epidemiologia, economia e sociologia. São Carlos: UFSCar, 2001.

AYRES, M.; AYRES JÚNIOR, M.; AYRES, D.L.; SANTOS, A. A. BIOESTAT - Aplicaçóes estatísticas nas áreas das ciências bio-médicas. Ong Mamiraua. Belém, PA. 2007.

BAUER, A.; DIEPGEN T. L.; SCHMITT J. Is occupational solar ultraviolet irradiation a relevant risk factor for basal cell carcinoma? A systematic review and metanalysis if the epidemiological literature. $\mathrm{Br} \mathrm{J}$ Dermatol. 165(3):612-25, 2011.

BATISTA FILHO, M. B.; MELO, M. N. T. Alimentaçáo, agrotóxicos e saúde. Rev. Bras. Saúde Mater. Infant. vol.12 n. ○2 Recife Abril/Junho 2012.

BOMBARDI, L. M. Intoxicação e morte por agrotóxicos no Brasil: a nova versáo do capitalismo oligopolizado. Bol. Dataluta. set;(45):1-21, 2011.

CALLE, E. E.; FRUMKIN, H.; HENLEY, S. J.; SAVITZ, D. A.; THUN, M. J. Organochlorines and breast cancer risk. A Cancer Journal of Clinicians. v. 52, n. 5, p. 301 - 309, 2002.

CARNEIRO, F. F.; PIGNATI, W.; RIGOTTO, R. M.; AUGUSTO, L. G. S.; RIZOLLO, A.; MULLER, N. M.; ALEXANDRE, V. P.; FRIEDRICH, K.; MELLO, M. S. C. Uma alerta sobre os impactos dos agrotóxicos na saúde. Dossiê ABRASCO, Rio de Janeiro, 2012.

COCCO, $\mathrm{P}$. On the rumors about the silent spring. Review of the scientific evidence linking occupational and environmental pesticide exposure to endocrine disruption health effects. Cadernos de Saúde Pública. Rio de Janeiro, v. 18, n. 2, p. 379 - 402, 2002.

DENNIS, L. K.; LYNCH, C.F.; SANDLER, D.P.; ALAVANJA, M. C. Pesticide use and cutaneous melanoma in pesticide applicators in the agricultural health study. Environ Health Perspect. 118(6):812-7, 2010.

DOLAPSAKIS, G.; VLACHONIKOLIS, I. G.; VARVERIS, C.; TSATSAKIS, A. M. Mammographic findings and occupational exposure to pesticides currently in use on Crete. European Journal of Cancer. v. 37, n. 12, p. 1531 - 1536, 2001. ETGES, V. E. O impacto da cultura do tabaco no ecossistema e na saúde humana. Textual, Porto Alegre, v.1 n.1, p. 14-21, nov, 2002.

FASSA, A. G.; FARIA, N. M. X.; MEUCCI, R. D.; FIORI, N. S.; MIRANDA, V. I.; FACCHINI, L. A. Green Tobacco 
Sickness Among Tobacco Farmers in Southern Brazil. Am J Ind Med; 57(6):223-300, 2014.

FRANCESCHI, L. V. C. Cancer epidemiology in the elderly. Critical Reviews in Oncology/Hematology. 39/ 219-226, 2001. GARCIA, E. G. Segurança e Saúde no trabalho rural com agrotóxicos: contribuiçáo para uma abordagem mais abrangente [dissertação]. São Paulo (SP): Faculdade de Saúde Pública, Universidade de São Paulo; 1996.

GAMMON, M. D.; WOLFF, M. S.; NEUGUT, A. I.; ENG, S. M.; TEITELBAUM, S. L.; BRITTON, J. A.; TERRY, M. B.; LEVIN, B.; STELLMAN, S. D.; KABAT, G. C.; HATCH, M.; SENIE, R.; BERKOWITZ, G.; BRADLOW, H. L.; GARBOWSKI, G.; MAFFEO, C.; MONTALVAN, P.; KEMENY, M.; CITRON, M.; SCHNABEL, F.; SCHUSS, A.; HAJDU, S.; VINCEGUERRA, V.; NIGUIDULA, N.; IRELAND, K.; SANTELLA, R. M. Environmental toxins and breast cancer Long Island. II. Organochlorine compound levels in blood. Cancer Epidemiology Biomarkers \& Prevention. v. 11, n. 8, p. $686-697,2002$.

IBGE - Instituto Brasileiro de Geografia e Estatística. Censo demográfico 2010: características gerais da população. Resultados da amostra. Disponível em: http://www.ibge.gov.br acesso em 31/10/2013.

INPE - Instituto Nacional de Pesquisas Espaciais. TERRAVIEW versão 4.2.2. Software disponível em: http://www.dpi.inpe.br/ terraview/index.php. São José dos Campos, SP. 2010.

JOBIM, P. F. C.; NUNES, L. N.; GIUGLIANI, R.; CRUZ, I. B. M. DA. Existe uma associacao entre mortalidade por cancêr e uso de agrotoxicos? Uma contribuicao ao debate. Ciência \& Saúde Coletiva. 15.1 p277, 2010.

KOIFMAN, S.; HATAGIMA, A. Exposição aos agrotóxicos e câncer ambiental. É Veneno ou É Remédio? - agrotóxicos, saúde e ambiente. p. 75-89, 2003.

LANSBURY L.; BATH-HEXTALL F. What's new in skin cancer? An evidence-based update. Dermatol Nurs. 11(1):33-
9, 2012

MOREIRA, J. C.; JACOB, S. C.; PERES, F. Avaliaçáo integrada do impactodo uso de agrotóxicos sobre a saúde humana em uma comunidade agrícola de Nova Friburgo, RJ. Ciência \& Saúde Coletiva 7(2): 299-311, 2002.

OLIVEIRA, P.P.V.; SIHLER, C.B.; MOURA, L.; MALTA, D. C.; TORRES, M. C. A.; LIMA S. M. C. P.; LIMA, A. L. A. DE.; LEITE, C. E.; SILVA, V. L. C.; SOBEL, J.; LANZIERI, T. M. First reported outbreak of Green tobacco sickness in Brazil. Cad Saúde Publica 26(12):2263-2269, 2010.

OPAS (Organização Pan-Americana da Saúde). Ministério da Saúde do Brasil. Secretaria de Vigilância Sanitária. Manual de Vigilância de populaçóes expostas a agrotóxicos. Brasília, DF; 1996.

RIQUINHO, D. L.; HENNINGTON, E. A. Tobacco cultivation in the south of Brazil: green tobacco sickness and other health problems. Ciência \& Saúde Coletiva. 19.12: p4797, 2014

SILVA, E. F.; PANIZ, V. M. V.; LASTE, G.; TORRES, I. L. $S$. Prevalência de morbidades e sintomas em idosos: um estudo comparativo entre zonas rural e urbana. Ciência \& Saúde Coletiva. 18(4): 1029-1040, 2013.

SILVA, F. S.; SILVA, A. M. C.; LUZ, L. L.; AYDOS, R. D.; MATTOS, I. E. Correlação entre produção agrícola, variáveis clínicas-demográficas e câncer de próstata: um estudo ecológico. Ciência \& Saúde Coletiva.20(9): 2805-2812, 2015. SOUZA, A.; MEDEIROS, A. R. SOUZA, A. C.; WINK, M.; SIQUEIRA, I. R.; FERREIRA, M. B. C.; FERNANDES, L.; HIDALGO, M. P. L.; TORRES, I. L. S. Avaliaçáo do impacto da exposição a agrotóxicos sobre a saúde de populaçáo rural. Vale do Taquari (RS, Brasil). Ciência \& Saúde Coletiva. 16(8): 3519-3528, 2011.

VEIGA, M. M. Agrotóxicos: eficiência econômica e injustiça socioambiental. Ciênc. saúde coletiva vol.12 n. ${ }^{\circ} 1$ Rio de Janeiro Jan./Mar. 2007. 